\title{
PRODUCTIVIDAD PRIMARIA DE TRES PRADERAS DE ESPECIES \\ TROPICALES: PARA (BRACHIARIA MUTICA), GRAMA AMARGA (PASPALUM CONJUGATUM) Y PANGOLA (DIGITARIA DECUMBENS)
}

\author{
RICARDO ALMEIDA M. ${ }^{\circ}$
}

\section{INTRODUCCION}

Un comportamiento estructural del ecosistema, que representa las plantas, tiene la función de fijar energía solar a través del proceso fotosintético. La productividad primaria se refiere a la energía fijada que sustenta la respiración y da origen a toda la materia orgánica del sistema. La productividad neta es el remanente de energía después de efectuar la respiración, dicha producción es por incrementos absolutos de biomasa.

El concepto productividad presenta varias modalidadse; rendimiento y cosecha son términos relacionadas con productividad neta, según la expresa Odum (1971); por otra parte, Montieth, (1965), emplea cifras de productividad neta e indica eficiencia de energía solar convertida en materia vegetal, deduce que al término del proceso fotosintético debe quedar una cantidad de energía después de la que es utilizada en el proceso mismo. El método de Odum sugiere la medición práctica de la biomasa presente, como un todo o específicamente la utilizable en forma de alimento. En cambio, Montieth sugiere recurrir a la fuente de energía y encontrar los lugares donde ésta se deposita. Singh (1974) al revisar los trabajos relativos a la productividad primaria encontró diferencias de resultados. Debido a la conceptualización y metodología, él mismo traduce los resultados en términos comunes.

En nuestro caso, productividad primaria hace referencia al crecimiento (G): es la única medida del total de la materia orgánica producida y consiste en el aumento de biomasa o su disminución, va que ambas son expresiones del material transferido v utilizado en el sistema. Estos conceptos empleados por Petrusewicz (1967a: 1967b) en productividad secundaria, son igualmente aplicables para productividad primaria.

Nuestro objetivo de estudio es emplear esquemas conceptuales que permitan integrar información complementaria al cálculo de productividad primaria; esto es, inteorar mediciones de los cambios de biomasa y estimaciones del material desprendido por la planta en el ciclo anual.

\section{MATERIALES Y METODOS}

\section{Descripción del lugar de estudio y materiales}

Los sistemas estudiados son tres praderas contiguas al Colegio Superior de Agricultura Tropical de Cárdenas, Tabasco, en latitud N $18^{\circ}$ y longitud E $94^{\circ}$

- Profesor Investigador del Colegio Superior de Agricultura Tropical. Apartado Postal 24. H. Cárdenas, Tabasco. México.

Almeida-M R. 1981. Productividad primaria de tres praderas de especies tropicales: Para (Brachiaria mutica), Grama amarga (Paspalum conjugatum) y Pangola (Digitaria decumbens). Boletín de la Sociedad Botánica de México 41: 3-18. 
30’. En estos lugares había Selva Alta Perennifolia (Miranda y Hernández X, 1959) y presenta un clima cálido húmedo, Am (f) w' (i') g, (García, 1973). La figura 1 muestra la temperatura y precipitación en sus medias mensuales durante el periodo de estudio.

\section{Las praderas}

El "pará" o "zacate egipto" (Brachiaria mutica) fue trasplantado a un área de 16 ha, una especie bastante apetecible, común en la zona y permanece en lugares inundados de tierras bajas. En la época de menos lluvia queda al descubierto este pasto y el ganado se "baja" a estas zonas. Al mismo tiempo fue trasplantado el "pangola" (Digitaria decumbens) en un área de 16 ha.

La pradera de "grama amarga" (Paspalum conjugatum) brotó al natural en 4 ha, donde se había efectuado el desmonte. El ganadero de Tabasco tiene grandes extenciones donde solo hay esta especie, siendo más común en zonas altas del estado.

Las praderas se iniciaron casi al mismo tiempo, marzo 1968. Nuestro estudio comenzó 18 meses después durante el periodo agosto de 1970, hasta agosto de 1971.

\section{Procedimiento}

Las diferentes praderas presentaban homogeneidad estructural, con escasas o ninguna otra especie entremezclada. En cada una de ellas se localizaron aleatoriamente 10 cuadros de $1 \mathrm{~m}^{2}$. El propósito fue colectar muestras de la biomasa de la parte aérea (BPA), de la biomasa de la raíz (BR) y de la hojarasca en el primer tiempo $(\mathrm{H}) \mathrm{t}_{6}$ y el segundo tiempo $\left(\mathrm{H}^{\prime}\right) \mathrm{t}_{0}+1$.

Las mediciones iniciales se hicieron cada dos meses, hasta diciembre para la "grama" y "pará" y hasta febrero para "pangola"; después los registros fueron mensuales.

La parte aérea verde (BPA) es la biomasa encontrada arriba del nivel del suelo y en crecimiento. Los márgenes de los cuadros dificultaban el corte deseado para el pará, ya que su hátio estructural es erecto y más alto que los otros dos. Los pesos fueron determinados en base seca.

Las raíces fueron extraidas de un cubo de tierra de $0.25 \mathrm{~m}^{2}$ de superficie con 25-30 $\mathrm{cm}$ de profundidad. Los terrenos fueron sumergidos en agua para facilitar el lavado de las partes visibles y recoger por flotación las raicillas que posteriormente fueron secadas para obtener su peso.

La hojarasca representa todo el material muerto yacente en el suelo, abajo de la parte aérea verde. En el "pará" estas partes muertas estaban prendidas a la planta y en menor grado en "pangola", las cuales fueron separadas y consideradas también como hojarasca expuesta a descomposición, sin la contribución de más hojas desprendidas. El último material se dejó de un mes a dos meses según el tiempo entre muestreos. Esto representa el material expuesto a descomposiciớn $\left(\mathrm{H}^{\prime}\right)$. 


\section{Cálculo de productividad}

La BPA define los niveles de biomasa en los diferentes tiempos de muestreo, y su disminución o aumento define los cambios.

Así la ecuación:

$$
\mathrm{PBPA}=\sum_{i=1}^{\mathrm{n}}\left(\Delta \mathrm{BPA}_{\mathrm{i}}\right)
$$

define la producción parcial de la parte aérea como resultado del balance de biomasa en el enésimo periodo de tiempo.

Por similitud, la biomasa de la raíz es:

$$
\operatorname{PBR}=\sum_{i=1}^{n}\left(\Delta \mathrm{BR}_{\mathrm{i}}\right)
$$

Tanto la BPA como la BR se sumaron y los cambios detectados fueron en base a la biomasa global (B).

La hojarasca se empleó de acuerdo al método de Weigert y Evans (1964) para calcular el material desprendido. El material se recogió en la forma anterior-

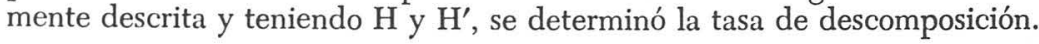

$$
t d=\frac{\ln \left(\mathrm{H} / \mathrm{H}^{\prime}\right)}{t_{0}+1-t_{0}}
$$

donde $\mathrm{H}$ representa las 10 muestras en $t_{0}$ y $H^{\prime}$ las muestras en $t_{0}+1$ expuestas a descomposición. El cociente es expresado en logaritmos naturales y se divide entre los días transcurridos entre muestreos.

El material desprendido (E) se calcula de la siguiente manera:

$$
E=\frac{H+H H}{2} \times t d \times t_{i}
$$

Donde $\mathrm{HH}$ es un dato de la hojarasca presente al finalizar el periodo de muestreo, pero a diferencia de $\mathrm{H}^{\prime}$ se tiene la contribución del material que va cayendo, ya que la BPA permaneció arriba de ésta. Entonces $\frac{\mathrm{H}+\mathrm{HH}}{2}$ indica el promedio de la hojarasca en tiempo $\mathrm{i}_{\mathrm{i}}$ expuesto a descomposición, lo que al multiplicarse por la tasa de descomposición estima el total del material desprendido en ese mismo periodo. Dado que el proceso de eliminación o desprendimiento de hoja es continuo, hemos de considerar que múltiples factores perturban la caída directa de hoja al suelo, además es removida por viento o inundaciones. 


\section{RESULTADOS Y DISCUSION}

\section{Praderas de pará}

El pasto pará presenta la mayor producción de materia orgánica $10,167 \mathrm{~g} / \mathrm{m}^{2}$ / año. La forma en que se obtuvo esta cifra se demuestra en los siguientes párrafos. En el cuadro 1 se presentan las cantidades de los pesos registrados y los valores medios se presentan gráficamente en la figura 1 . Los componente de BPA, BR y E muestran diferentes niveles durante el año de estudio. La BR y E son multiplicados por 4 para dar correspondencia a la expresión por metro cuadrado. En BPA y $\mathrm{BR}$ son valores medios y su variabidad se expresa en barras verticales, correspondiendo al error estándar de cada media, e. g. el peso medio de 10 muestras para agosto es de 74.44 gramos/m² y su error estándar es 19.09.

La eliminación o desprendimiento de hoja (E) forma otro componente y se calcula de los diferentes niveles de hojarasca (Weigert y Evans, 1964). Primero se determina la tasa de descomposición; así, para calcular E consideramos el periodo del 14 al 12 de diciembre, 59 días.

$$
\mathrm{td}=\frac{\ln (642 / 134,92)}{59}=.026
$$

El material expuesto a esa tasa de descomposición calculada, es el promedio de hojarasca existente en el inicio de un periodo y al final del mismo; para el periodo considerado anteriormente:

$$
\begin{aligned}
& \text { Material expuesto } \\
& \text { a a } \\
& \text { descomposición }
\end{aligned}=\frac{642.2+838.96}{2}=740.58
$$

Entonces:

$$
\mathrm{E}=740.58 \times 0.26 \times 59=1136
$$

En el caso particular del pará, algunas de las cantidades de hojarasca fueron colectadas del desprendimiento de partes muertas en el tallo y algunas veces las inundaciones acarreabas todo el material orgánico sobre el suelo. El material desprendido (E) en el año es la suma de las cantidades calculadas para cada periodo.

$$
\mathrm{E}=\frac{\mathrm{n}=10}{\mathrm{i}=\frac{1}{1}} \mathrm{E}_{\mathrm{i}}
$$

En la figura 2 se muestra el total de E, siendo 8,529 gramos por año por metro cuadrado. Las cantidades de BPA y BR se han sumado; así 74.44 y 504 representan 578 gramos de biomasa en el mes de agosto 11, 1970. En los periodos que hubo una diferencia positiva de biomasa, se consideró como ganancia (g); así, de agosto 11 de octubre 14, la ganancia fue:

$$
\mathrm{g}=578-868.08=90.08
$$


Cuadro 1. Peso seco de biomasa de la parte área, biomasa, raíz y hojarasca del pará (Brachiaria mutica) en el periodo de agosto 1970, a agosto 1971. H. Cárdenas, Tabasco.

$\begin{array}{lcccccc} & \begin{array}{c}\text { Biomasa } \\ \text { parte área } \\ \text { g.m }{ }^{-2}\end{array} & \text { E.S. }{ }^{*} & \begin{array}{c}\text { Biomasa } \\ \text { raíz } \\ \text { g.m }{ }^{-2}\end{array} & \text { E.S. }{ }^{*} & \text { H } & \mathrm{H}^{\prime} \\ \text { Agosto 11, 1970 } & 74.44 & 19.09 & 504 & 53.29 & 606.00 & \\ \text { Octubre 14 } & 373.16 & 51.57 & 494.92 & 70.56 & 642.2 & 53.92 \\ \text { Diciembre 12 } & 796.24 & 44.03 & 451.2^{\circ \circ} & 36.06 & 838.96 & 134.92 \\ \text { Enero 14, 1971 } & 511.92 & 42.76 & 233.52 & 29.14 & 607.2 & 215.44 \\ \text { Febrero 17 } & 556.92 & 21.16 & 523.2 & 57.24 & 758 & 136.72 \\ \text { Marzo 18 } & 540.16 & 74.3 & 321.52 & 34.36 & 828.6 & 257.2 \\ \text { Abril 17 } & 587.52 & 48.31 & 521.92 & 57.08 & 824.32 & 262.16 \\ \text { Mayo 18 } & 654.68 & 44.68 & 382.88 & 43.4 & 1052.5 & 190.56 \\ \text { Junio 21 } & 761 & 32.45 & 498.76 & 31.98 & 1067.76 & 265.68 \\ \text { Julio 20 } & 1067.4 & 55.69 & 381.84 & 39.80 & 1271.28 & 101.12 \\ \text { Agosto 16 } & 769.88 & 58.42 & 206.68 & 32.68 & 1141.69 & 160.2\end{array}$

- Error estándar, $\mathrm{n}=10$.

$\therefore$ Estimación. 


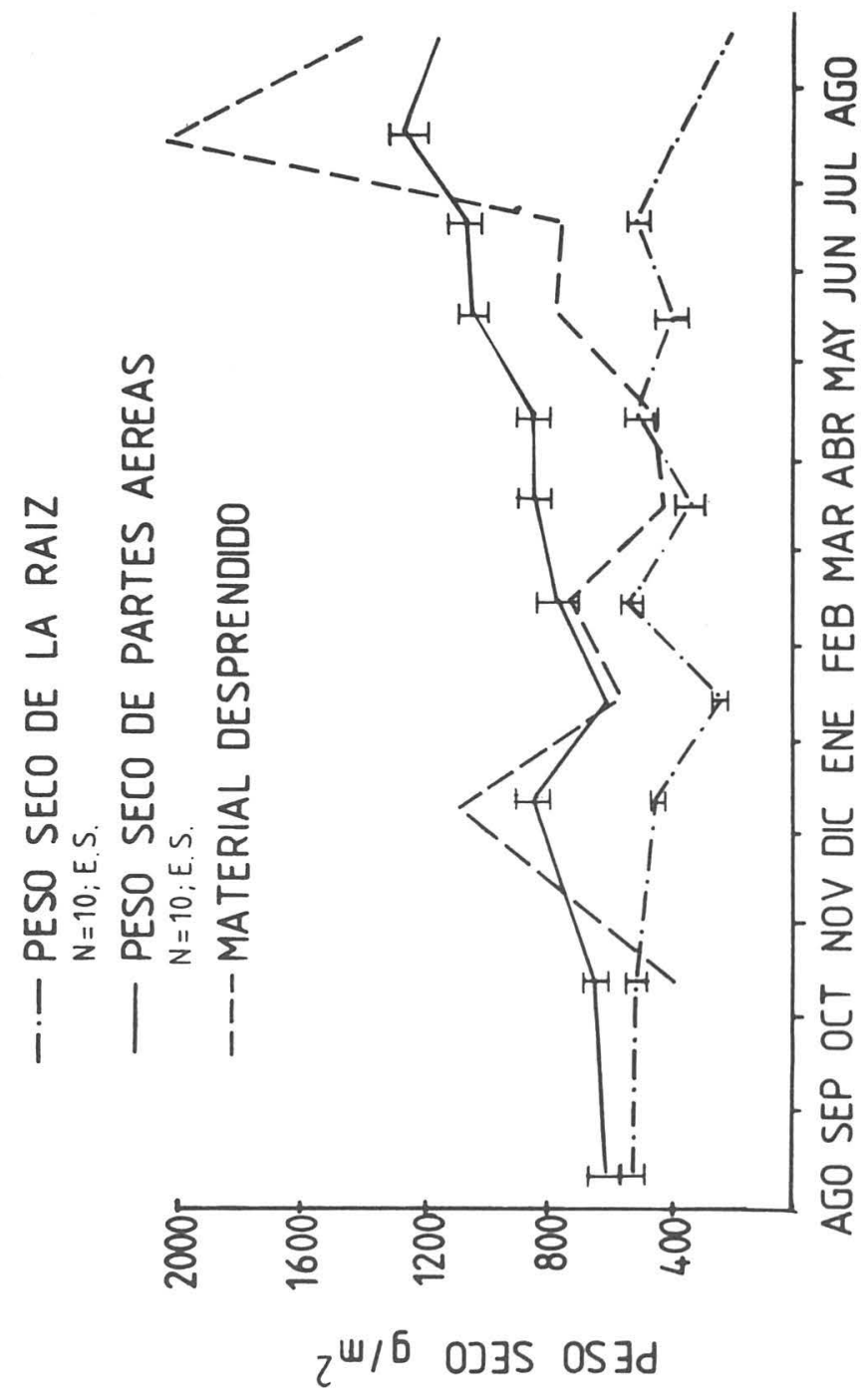

Figura 1. Distribución de peso de las diferentes estructuras de Brachiaria mutica, 1970-1971. 


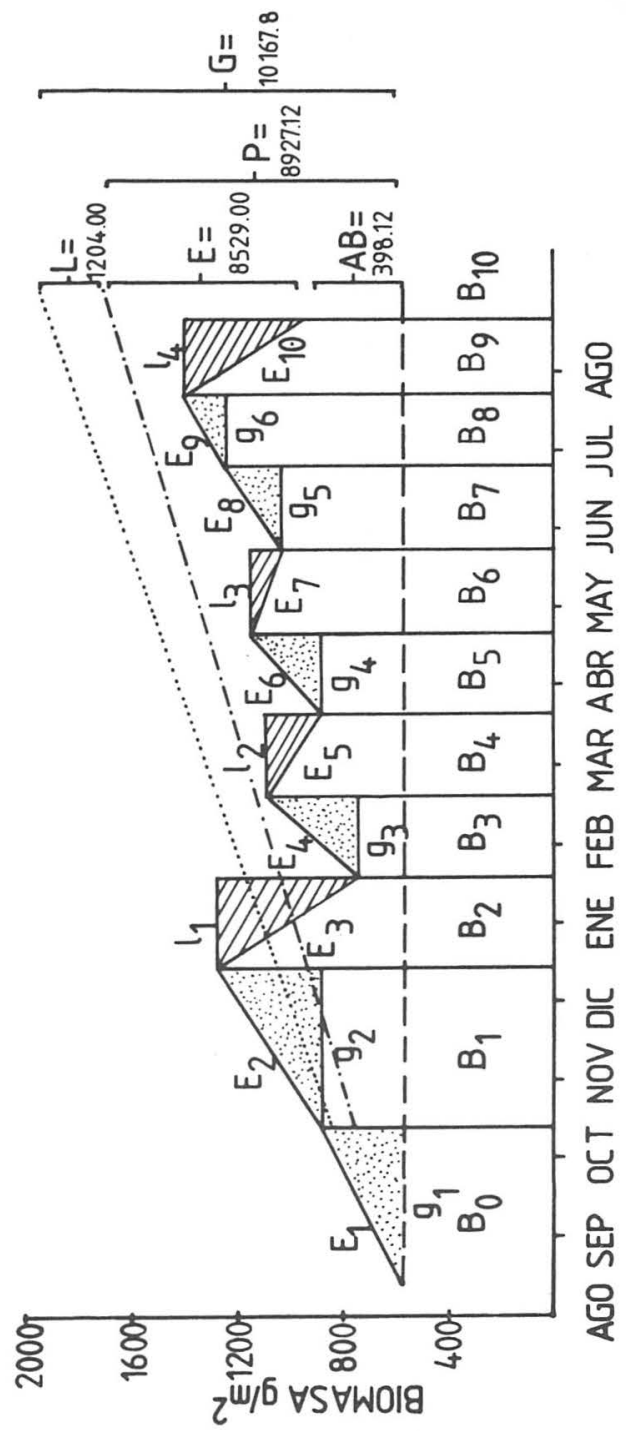

Figura 2. Cambios de biomasa y productividad de Brachiaria mutica, 1970-1971. 
En la figura 2 se muestra en forma resumida los diferentes niveles de biomasa; los que representan ganancias hacen un total $(\mathrm{G})$; donde sólo hubiera calculado $\mathrm{E}$, la producción observable incluiría el material orgánico total producido. Pero en los casos donde hay pérdidas de biomasa el cálculo es diferente, como se ha de demostrar enseguida.

Las pérdidas de biomasa, diferencias negativas (1) se suman y dan como resultado el total de materia orgánica perdida o no observable, dado que es consumida por el propio organismo para su mantenimiento, así tenemos:

$$
\mathrm{L}=\frac{\mathrm{n}=4}{\mathrm{i}=\frac{1}{1}}=1,204
$$

Entonces:

Donde

$$
\mathrm{B}+\mathrm{E}=\mathrm{P} \text { y } \mathrm{P}+\mathrm{L}=\mathrm{G}
$$

$$
\mathrm{P}=\text { producción o productividad neta }
$$

$\mathrm{G}=$ producción total de materia orgánica, incluyendo aquella biomasa

Praderas de grama amarga

La producción de materia orgánica de grama amarga en el año fue la menor, $808 \mathrm{~g} / \mathrm{m}^{2} /$ año. La forma en que se estimó esta cifra es similar a la anterior, en caso contrario se harán las aclaraciones correspondientes, pero todas las estimaciones se han conceptualizado en los modelos de Petrusewicz (1967a; 1967b).

En el cuadro 2 se presentan las cifras registradas, valores medios, error estándar y pesos de hojarasca. En la figura. En la figura 3 se observan los diferentes niveles en los diferentes meses del año. En la figura 4 se resume y se muestran las contribuciones por componente. En este caso el nivel de biomasa final muestra una cantidad menor que la inicial, donde $\mathrm{L} O$ y entonces $\mathrm{G}=\mathrm{P}+\mathrm{L}$. La eliminación (e) es un estado intermedio entre productividad neta y cambio de biomasa, siendo esta situación diferente a la de pará, donde el nivel final fue mayor al inicial en el periodo anual. Los valores de L, E, P y G fueron determinados de igual manera que el caso anterior.

También en la figura 4 se pueden observar las tendencias de conservación de $B$ en contraste con pará, donde la dirección de cambio negativo es opuesto. La grama presenta disminución de $\mathrm{B}$ de agosto, 1970 a enero, 1971 y de enero a mayo, 1971; el resultado de cambio es a un aumento drástico y finalmente de junio a agosto, 1971 la tendencia es a disminuir. El lugar donde permaneció la grama estuvo bien drenado y no se presentó infestación de "salivazo", caso contrario en el pará.

Praderas de pagola.

La producción de materia orgánica de pangola fue de 5,120 gramos por metro cuadrado por año. Los cálculos son similares a los anteriores, en caso contrario se hacen las aclaraciones respectivas.

En el cuadro 3 se presentan los pesos registrados. En la figura 5 se muestran los niveles de biomasa y hojarasca en los diferentes meses del año. En la figura 6 
Cuadro 2. Peso seco de la biomasa de la parte área, biomasa de la raíz y hojarasca de grama amarga (Paspalum conjugatum) durante el periodo de agosto 1970 - agosto 1971.

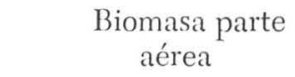

peso seco

g.m $\mathrm{m}^{-2}$

E.S.

peso seco

g. $\mathrm{m}^{-2}$

Biomasa

raíz
Hojarasca

$\mathrm{H}$

$\begin{array}{lcclccr}\text { Agosto 11, 1970 } & 445.52 & 42.16 & 920 & 106.04 & 179.24 & \\ \text { Octubre 14 } & 244.24 & 25.12 & 578.88 & 77.32 & 61.84 & 54.04 \\ \text { Diciembre 12 } & 459.48 & 85.96 & 624 & 85.02 & 178 & 51.24 \\ \text { Enero 15, 1971 } & 632 & 69.24 & 261.16 & 16.36 & 221.96 & 94.44 \\ \text { Febrero 17 } & 1000.6 & 59.54 & 512.76 & 68.24 & 220.24 & 123.88 \\ \text { Marzo 18 } & 965.64 & 98.08 & 302.96 & 33.8 & 229.26 & 164.36 \\ \text { Abril 17 } & 1276.32 & 86.88 & 576.76 & 80.12 & 259.52 & 196.96 \\ \text { Mayo 18 } & 1459.32 & 79.52 & 510.56 & 49.2 & 412.35 & 230.72 \\ \text { Jumio 21 } & 1169.84 & 35.08 & 352.92 & 36.44 & 425.6 & 327.92 \\ \text { Julio 20 } & 1102.16 & 105.8 & 341.04 & 29.8 & 794.24 & 374.32 \\ \text { Agosto 16 } & 908.16 & 42.96 & 293.52 & 33.0 & 559.76 & 462.32\end{array}$

- Error estándar, $\mathrm{n}=10$. 
Cuadro 3. Peso seco de biomasa de la parte área, biomasa de la raíz y hojarasca del pandola (Digitaria decumbens) durante el periodo de agosto 1970, agosto 1971. H. Cárdenas, Tabasco.

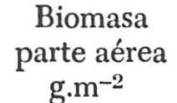

$$
\text { g.m }{ }^{-2}
$$

Biomasa

$$
\text { Biomasa }
$$

raíz

E.S. ${ }^{\circ} \quad$ g.m $\mathrm{m}^{-2}$

$54.96 \quad 1331.6$

$144.56 \quad 1477.76$

$72.32 \quad 999.2$

$62.08 \quad 908.28$

$36.32 \quad 746.96$

63.16

986.48

896.8

920.6

715.76

$1455.64 \quad 133.68$

1494.24

49.36

655.44

33.8

73.2
E.S. ${ }^{\circ}$

Hojarasca

$\mathrm{H} \quad \mathrm{H}^{\prime}$

g.m ${ }^{-2}$

g.m ${ }^{-2}$

$151.88 \quad 722$

$\begin{array}{lll}92.6 & 316.4 & 597.52\end{array}$

$\begin{array}{lll}69.35 & 603.76 \quad 128.28\end{array}$

$\begin{array}{lll}62.76 & 718.12 & 132.16\end{array}$

$\begin{array}{lll}70.12 & 222,36 & 148.36\end{array}$

120.0

257.44

189.95

Mayo 18

1121.22

60.08

268.44

143.68

Junio

1120.88

57.96

428.4

275.

Julio

Agosto 16

- Error estándar, n $=10$. 
Cuadro 4. Diferentes valores de productividad primaria en ambientes tropicales.

Vegetación

Productividad neta $\mathrm{g} / \mathrm{m} / \mathrm{año}$

Pradera (Kurushketra India) ${ }^{1}$

Bermuda (Cynodon dactylon)

Caña de azúcar²

Rangos de valores reportados para praderas tropicales ${ }^{3}$

1 Singh y Yadova, 1974.

2 Odum, 1971.

3 Golley y Lieth, 1972.

+ Cambellas, et al, 1972. Sólo los días de rápido crecimiento.

++ Considerando sólo los días de rápido crecimiento.

${ }^{++}$Considerando un promedio de $500 \mathrm{~g}^{-2} \mathrm{año}^{-1}$.
Producción total de material orgánica $\mathrm{g} / \mathrm{m}^{2} /$ año.
8.529

371

4,308

3,538

10,162

808

5.120

9.69

$8.9-11.2^{+}$

$67.8^{++}$

200-2000 


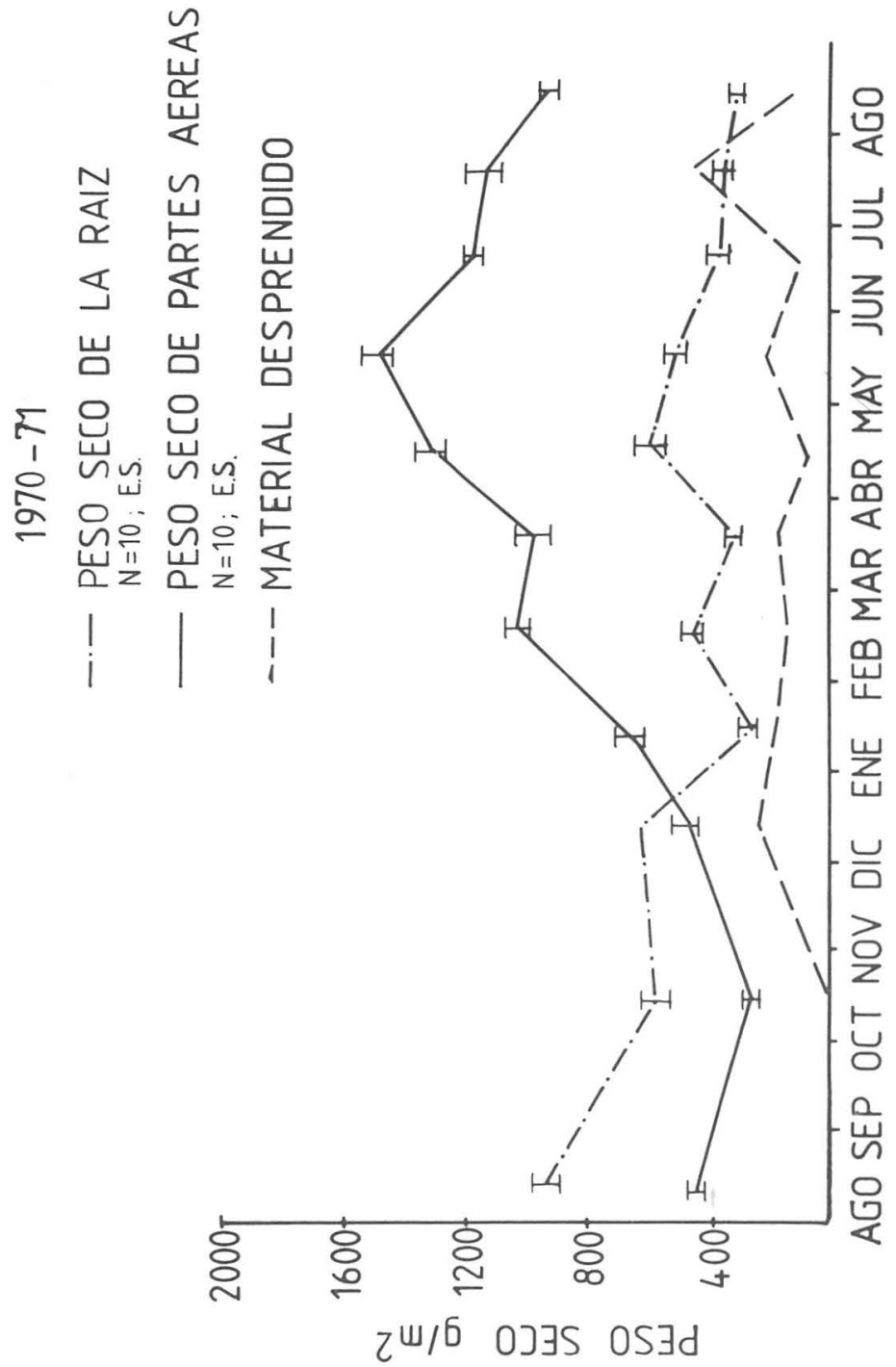

Figura 3. Distibución de peso de las diferentes estructuras de Paspalum conjugatum, 1970-1971. 


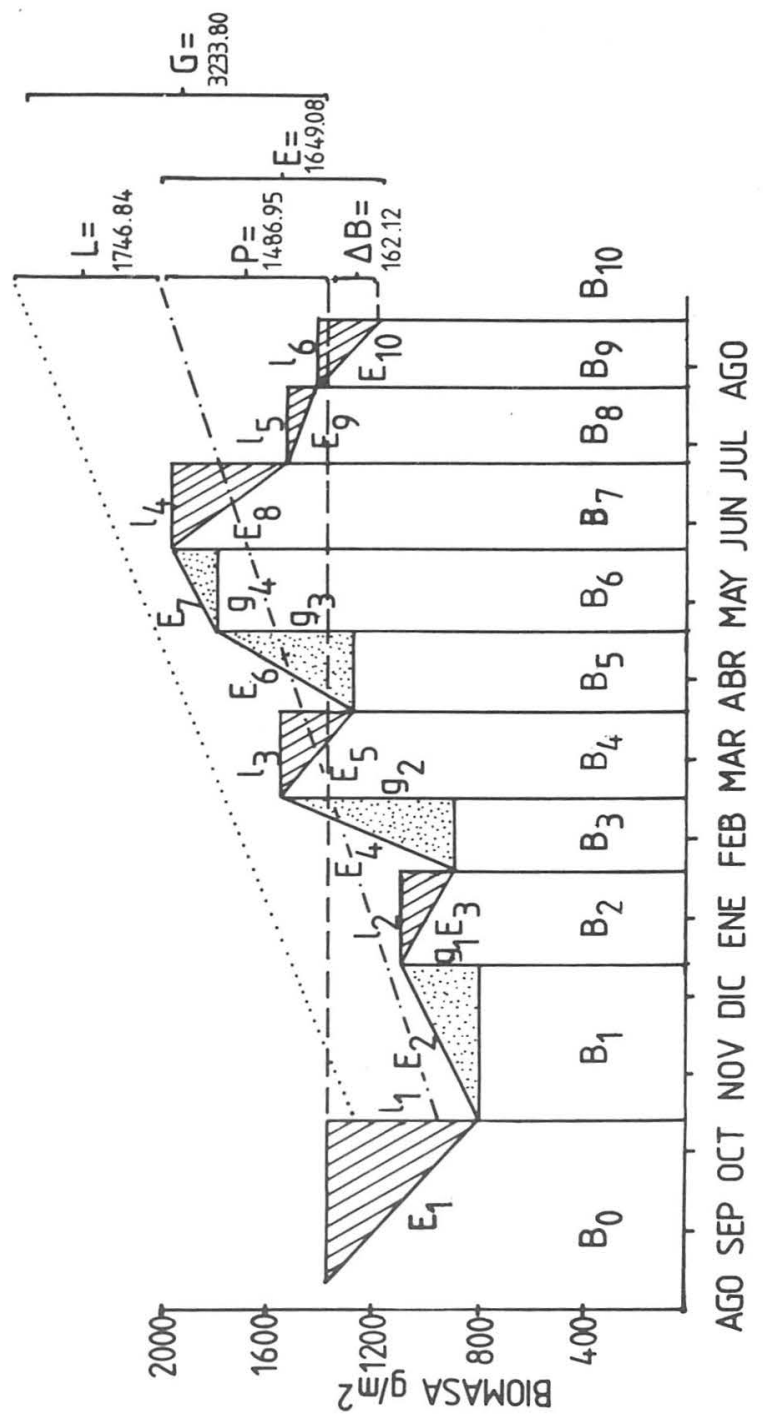

Figura 4. Cambios de biomasa y productividad de Paspalum conjugatum, 1970-1971. 


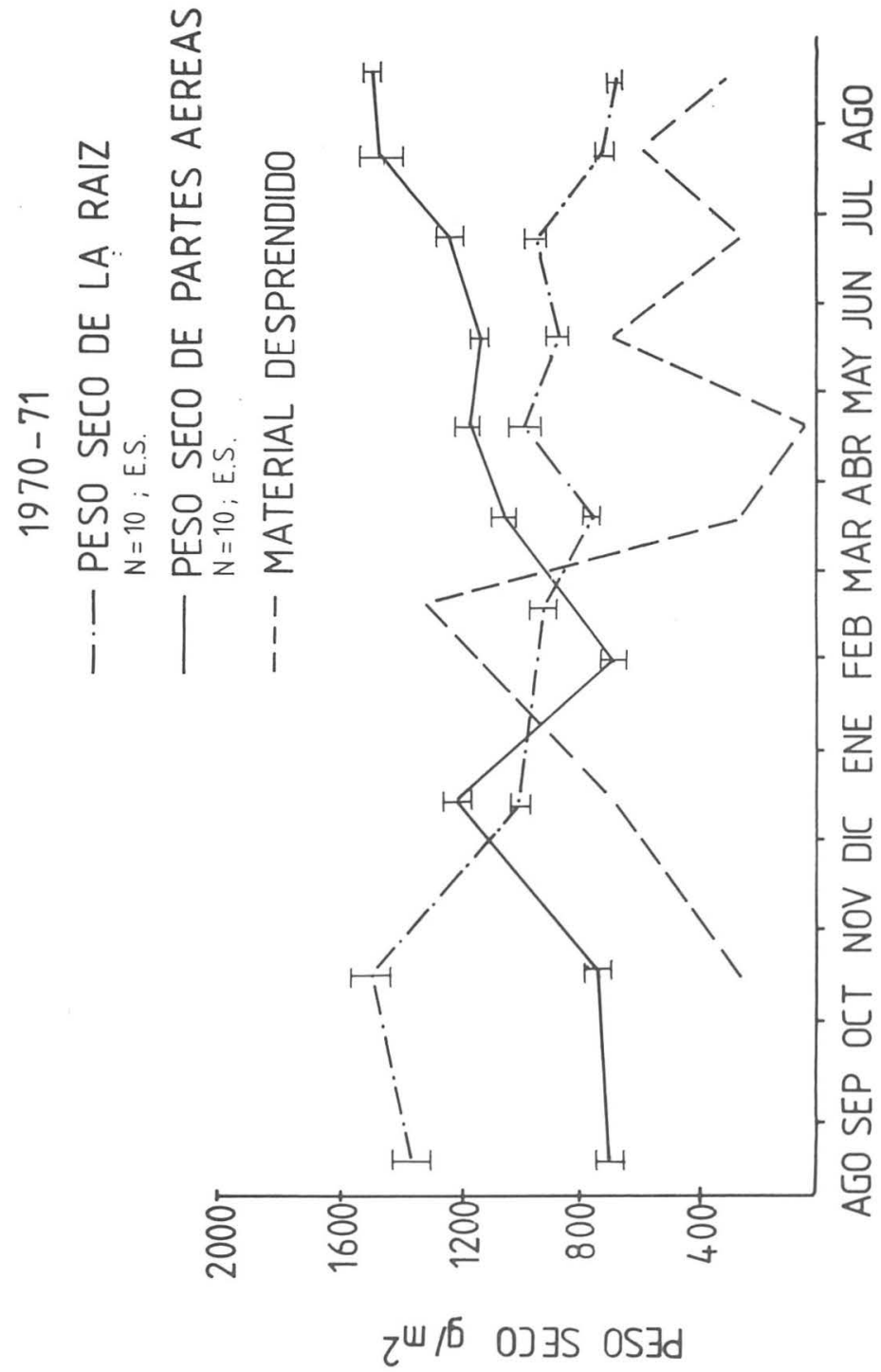

Figura 5. Distribución de peso de las diferentes estructuras de Digitaria decumbens, 1970-1971. 


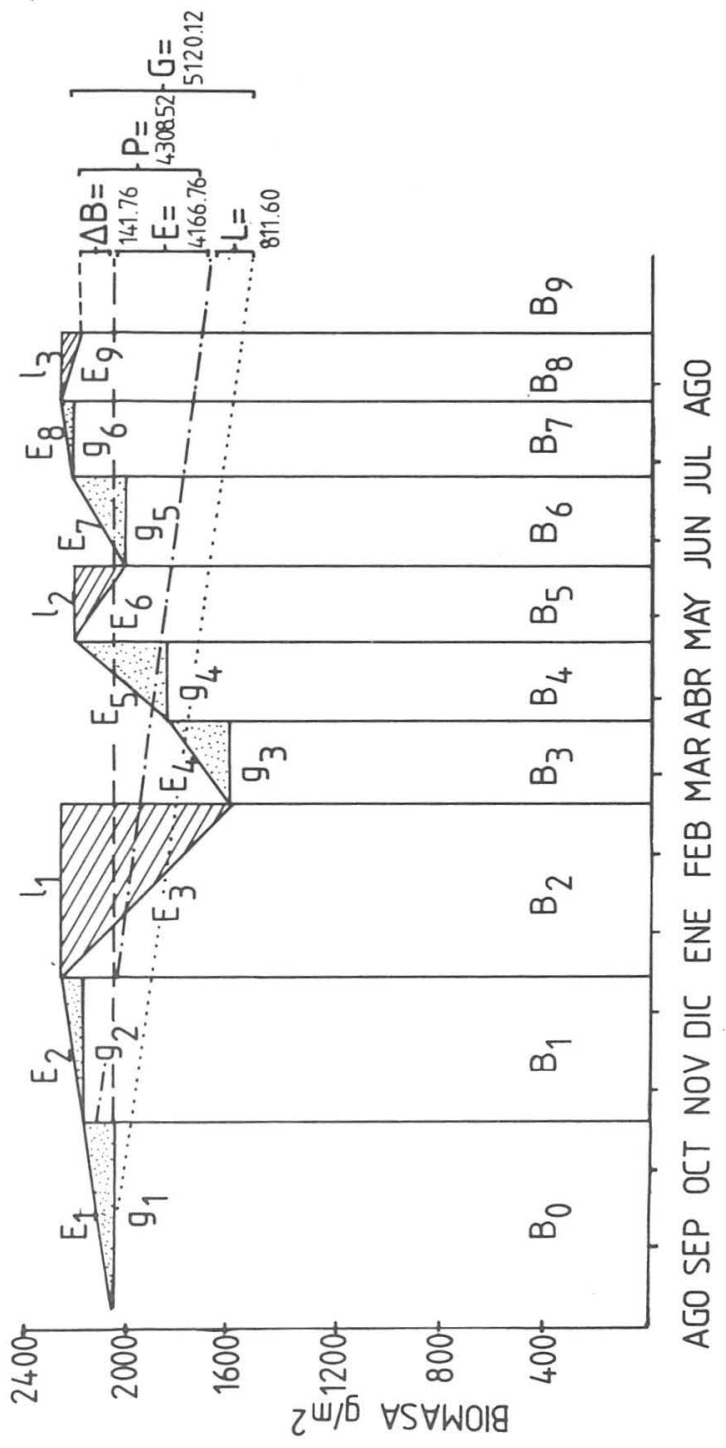

Figura 6. Cambios de biomasa y productividad de Digitaria decumbens, 1970-1971. 
resumen las contribuciones parciales a sus totales. La producción global es casi la mitad de la observada en pará durante el ciclo anual. Por otra parte, la grama; con parecidos niveles de biomasa que el pará, produjo 10 veces menos la cantidad global; siendo 10,167 y $808 \mathrm{~g} / \mathrm{m}^{2} /$ año, respectivamente. La productividad neta del pangola, estimada con la suma del material desprendido (E) y cambio positivo del nivel inicial en relación al nivel final anual, es de valor inferior, siendo este de $4,308 \mathrm{~g} / \mathrm{m}^{2} /$ año. El pangola muestra una ventaja de valores al conservar un colchón de materia orgánica muerta sobre el suelo, es posible que así garantice mayor estabilidad y abastecimiento de nutrientes durante el año.

Comparación de valores.

En la literatura se encuentran expresiones referidas a la producción neta y hay estimaciones sobre la productividad bruta o crecimiento total. En el cuadro 4 se observan valores además de los mencionados.

\section{BIBLIOGRAFIA}

CAMBELLOS, J.; E. GONZALES; A. TRUJILLO. Rendimiento y valor nutritivo de forrajes tropicales, 1. Bermuda Cu. Coastal, Cynodon dactylon (L) Pers. Agron. Trop. 22 ( 3 ): 321-238. 1972.

GARCIA, E. Modificaciones al sistema de clasificación climática de Kopen. Inst. de Geografía, UNAM. 1973.

GOLLEY, F.B.; H. LEITH. Bases of Organic Production in the Tropics. In: P. Golley y F.B. Gollcy (Eds) Symposium "Tropical Ecology with Emphasis on Organic Productivity. 1-125 pp. Georgia Athens (EE.UU.) 418 pp. 1972.

MIRANDA, F.; E. HERNANDEZ, X. Clasificación de los tipos de vegetación de México aplicable a los levantamientos forestales. (Reimpreso - Bol. Soc. Bot. Mex. 28: 27-179. 1963). 1959.

MONTIEH, J.L. Light distribution and photosynthesis in field crops. Ann. Bot. N.S. 29: 17-37. 1965.

ODUM, E.P. Fundamentals of Ecology. W.B. Saunders 574 pp. 1971.

PETRUSEWICZ, K. Suggested list of more important concepts in productivity studies (definitions and symbols) In K. Petrusewicz (Ed) Secondary productivity of Terrestrial ecosystems. Vol. 1. 51-58. Inst. Ecol. Polish Acad. Science. Varsovia, 879 pp. 1967a.

PETRUSEWICZ, K. Concepts in studies no the secondary productivity of terrestrial ecosystems. In: K. Petrusewicz (Ed) Secondary productivity of Terrestrial ecosystems. Vol. 1. 17-49. Inst. Ecol. Polish Acad. Science, Varsovia, 879 pp. $1967 \mathrm{~b}$.

SINGH, I.S.; D.S. YADAVA. Seagonal variation in composition nlant biomass, and net primary productivity of tropical grassland at Kurukshetra, India. Ecol. Monogr. 44: 351-376. 1974.

WEIGERT, R.G.; F.C. EVANS. Primary production and the disappearance of dead vegetation on an old field in south-eastern Michigan. Ecology 45: 49-53. 1964. 